\title{
Design and Control of 6-DOF Robotic Manipulator Driven by Pneumatic Muscles and Motor
}

\author{
Lian-Wang Lee, ${ }_{1}^{1}$ Liang-Yu Lu, ${ }^{2}$ I-Hsum Li, ${ }^{3}$ Chia-Wei Lee, ${ }^{1}$ and Te-Jen $\mathrm{Su}^{4}{ }^{4 *}$ \\ ${ }^{1}$ Department of Mechanical Engineering, National Chung Hsing University, \\ No. 145, Xingda Road, South District, Taichung City 40227, Taiwan, R.O.C. \\ ${ }^{2}$ Department of Mechanical Engineering, National Sun Yat-sen University, \\ No. 70, Lien-hai Road, Kaohsiung 80424, Taiwan, R.O.C. \\ ${ }^{3}$ Department of Mechanical and Electro-Mechanical Engineering, Tamkang University, \\ No. 151, Yingzhuan Road, Tamsui District, New Taipei City 25137, Taiwan, R.O.C. \\ ${ }^{4}$ Department of Electronic Engineering, National Kaohsiung University of Science and Technology, \\ No. 415, Jiangong Road, Sanmin District, Kaohsiung City 807618, Taiwan, R.O.C. \\ ${ }^{5}$ Graduate Institute of Clinical Medicine, Kaohsiung Medical University, Taiwan, R.O.C. \\ No. 100, Shih-Chuan 1st Road, Sanmin Dist., Kaohsiung City 80708, Taiwan
}

(Received December 30, 2020; accepted April 7, 2021)

Keywords: serial manipulator arm, smooth control, pneumatic-muscle actuator, linear extended state observer, novel proxy-based sliding mode control

A pneumatic-muscle actuator (PMA) is made of a unique material with fibers wrapped inside and metal fittings attached at each end. Because of reversible physical deformation, a PMA can produce linear motion during contraction and muscle expansion. We use a PMA and motors' hybrid drivers to implement a low-cost and safe robot manipulator with six degrees of freedom (6-DOF). Safety is achieved by applying a novel proxy-based sliding mode controller (NPSMC) and a linear extended state observer (LESO) on each joint of the 6-DOF robot manipulator. The NPSMC can compensate the six joints of the 6-DOF robot manipulator reaching Lyapunov stability, and we prove that their pathing errors converge to a neighborhood of zero. An experiment on all joints is conducted to verify the trajectory pathing precision and system safety for the 6-DOF robot manipulator. The experimental results show that under NPSMC compensation and the LESO estimate, the 6-DOF robot manipulator using the hybrid drivers satisfies both the pathing performance demands and safety control.

\section{Introduction}

In order to boost production efficiency and cut costs, a growing number of robot manipulators have been applied to production lines. Most robot manipulators on the market are driven by motors featuring high stiffness. High stiffness causes the robot arm to be unable to comply with the contact object when it encounters it, resulting in an unsafe impact force and poor flexibility; the better the flexibility, the better it can contact objects and avoid damage to the arm. Consequently, in recent years, some scholars have begun to study the development of lightweight,

*Corresponding author: e-mail: sutj@nkust.edu.tw

https://doi.org/10.18494/SAM.2021.3250 
low-energy-use, and low-cost robot manipulators with high flexibility and a simple structure, which can avoid the damage caused by loss of control in the case of breaking wires or the malfunction of sensors. The flexibility of a robot manipulator can be boosted in a passive or active manner. In the former case, high flexibility is achieved via flexible materials. A remote compliance center (RCC) made of six springs was the earliest flexible mechanism to be developed. ${ }^{(1)}$ In recent years, pneumatic muscles have been a research target; compared with traditional actuators, they have the merits of high flexibility, high carrying capacity, high power ratio, and a light weight. With an eye to safety, Ohta et al. ${ }^{(2)}$ designed a pneumatic-muscle robot manipulator with seven degrees of freedom (DOF), resorting to passive flexibility to alleviate the arm's impact upon contact with the environment. However, a momentary high-speed movement of the robot manipulator cannot be avoided in the case of a large position error. It turns out that passive flexibility is only applicable to special conditions and cannot prevent a robotic manipulator's unsafe impact when there is a large position error. Therefore, some scholars have proposed active flexibility from the perspective of control. Hogan and others, ${ }^{(3-5)}$ for instance, proposed impedance control, with the impedance parameters of a system adjusted to change its flexibility, thereby making the robot manipulator compliant with the environment. As a result, even in the case of a large error, the robot manipulator can remain flexible via feedback gain, although this entails more significant noise signals. Ho et al. ${ }^{(6)}$ used artificial neural training via an inverse dynamic model to realize flexible control. Given the high cost of a multi-axial robot manipulator, they installed a force sensor only at the terminal position, leaving out singular points. Japanese scholars Kikuuwe and coworkers ${ }^{(7,8)}$ presented proxy-based sliding mode control (PSMC) that realizes the same level of accuracy as proportional-derivative-integral control as long as the actuator is not saturated, but produces a smooth, overdamping resuming motion from large positional errors after the saturation of the actuator. In addition, chattering of the sliding mode control was presented using a continuous function and approximate discontinuous sign function. Upon contact with the environment, the system employed a saturation phenomenon to make the robot manipulator compliant with objects in contact. After the external resistance was eliminated, the system could carry out dynamic recovery with overdamping motion. Subsequently, Van Damme et al. ${ }^{(9)}$ applied PSMC in a pneumatic-muscle actuator (PMA) for a two-axial robot manipulator, attaining good safety control. Çandır ${ }^{(10)}$ then proposed a disturbance estimator for estimating the external disturbance to a system and increasing the robustness and precision of PSMC, attaining a good pathing effect. The only deficiency of PSMC is its incomplete stability theory. Kikuuwe ${ }^{(11)}$ proposed a proof for PSMC stability via a non-smooth Lyapunov method. However, it is only applicable to one-dimensional space and cannot prove the stability of machines in operation. Ding et al. ${ }^{(12)}$ presented a system model of a model proxy-based sliding mode controller (MPSMC), proving its stability with Lyapunov theory. An experiment showed that an MPSMC could ensure PSMC safety but is inconvenient in application owing to the need for the system model's actual parameters. In this study, we propose a linear extended state observer (LESO) based on MPSMC control theory capable of estimating the total disturbance (unknown parameters and internal and external disturbances) to overcome the difficulty of modeling. Early on, $\operatorname{Han}^{(13)}$ presented a nonlinear extended state observer (NLESO) and estimated the system's total disturbance. However, it is 
not easy to adjust the NLESO parameters. Therefore, Gao and coworkers ${ }^{(14-17)}$ proposed a LESO, and at the same time proposed the meaning and adjustment method of the parameters in the LESO, which simplifies the control algorithm and is widely used in various fields. We took advantage of the merits of a PMA, namely its light weight, high power ratio, and large output force, in designing a 6-DOF serial robotic manipulator driven by the combination of a flexible pneumatic muscle and a motor, which is coupled with a new MPSMC and LESO, thus boosting the robustness, compliance ability, and safety of the system. In the case of a significant position error, it ensures the robot manipulator's dynamic recovery to the expected trajectory via overdamping motion. Under normal conditions, the system can achieve target pathing based on error. Double mechanisms can be installed in the robot manipulator's hardware and software to enhance its safety and flexibility.

\section{System Structure}

We designed a 6-DOF serial robot manipulator equipped with a PMA, having the features of light weight, low cost, easy assembly, high power ratio, and good compliance ability, as shown in Fig. 1. The robot manipulator consists of three sections, namely, a shoulder (SH), elbow (E), and wrist (W), each capable of rotating freely via a pair of axes counteracting and pulling against each other, where $y_{1}-y_{5}$ represent the measurement angles and $Y$ and $Z$ are the turning directions of each axis. The experimental machine consists of a PMA single-axis system, a joint system, and a control system, whose structures and major components are introduced briefly in the following. The PMA single-axis system mainly consists of a pair of PMAs, a couple of proportional pressure-regulating valve modules, a rotary encoder, a circuit, and an air channel, as shown in Fig. 2. The PMA single-axis system employs compressed air as the motive force and

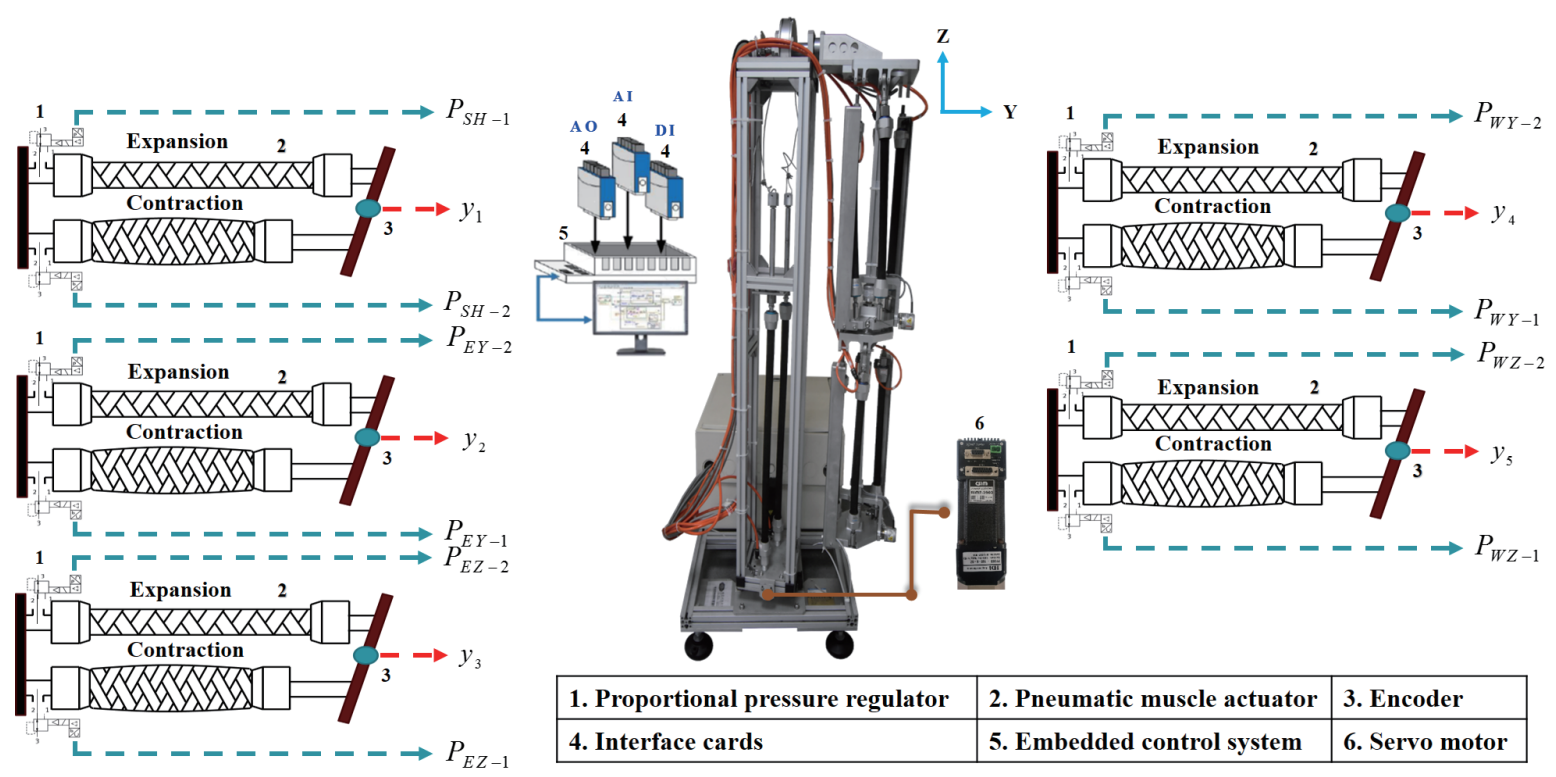

Fig. 1. (Color online) Photograph of 6-DOF robotic manipulator with hybrid drive. 


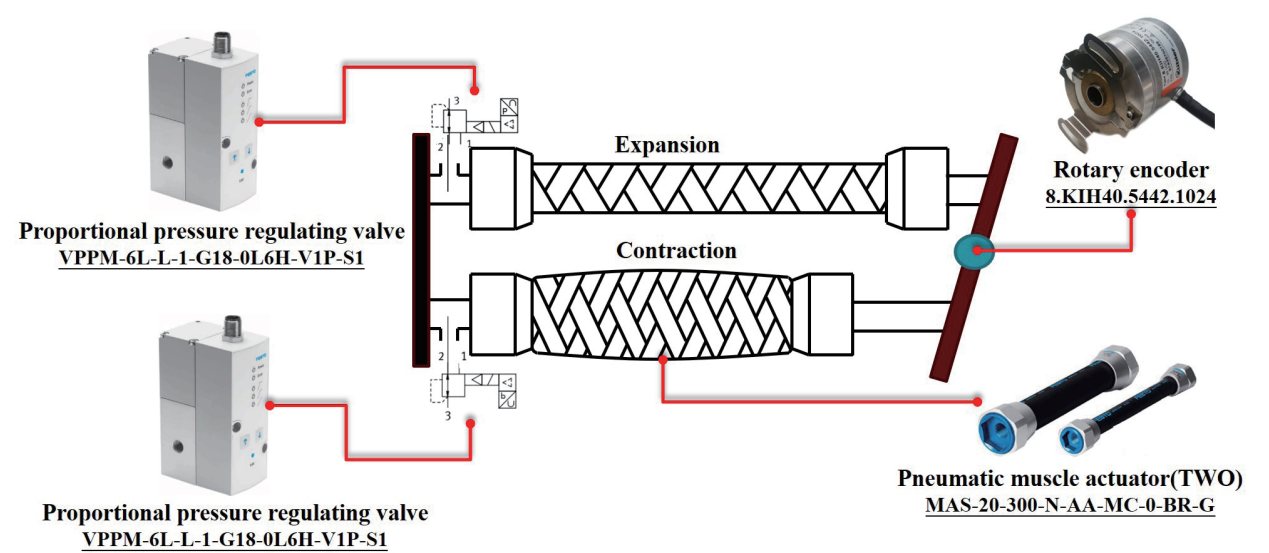

Fig. 2. (Color online) PMA single-axis system.

an embedded control system featuring a self-developed LabVIEW control program, an A/O interface for adjusting the pressure output of the pressure-regulating valve to control the outstretch and drawback of the robot manipulator, and a D/I interface to receive the feedback angle of the rotary encoder. The robotic-arm joint consists of a shoulder, elbow, and wrist; each joint comprises at least one pair of antagonistic PMAs. The shoulder is equipped with a pair of FESTO PMAs (model MAS-20-600-N-AA-MC-0-BR-G). A wire rope is used to manipulate the upper rotary device, which results in 1-DOF back and forth motion, as shown in Figs. 3(a) and 3(b). The elbow and wrist are equipped with two pairs of FESTO PMAs (model MAS-20-300-NAA-MC-0-BR-G). Wire ropes are used to drive the rotating turntable and the wire rope platform to achieve 2-DOF rotation and swing motion, with jigs employed to fix the muscles, solving the problem of mutual interference by the two pairs of PMAs during counteracting and pulling, as shown in Figs. 4(a) and 4(b). An NI Compact RIO forms the core of the control system, which employs an A/O interface card, A/I interface card, and an encoder, with LabVIEW visual programming for the retrieval, conversion, and decoding of I/O signals, enabling real-time control of the multijoint robot manipulator.

\section{Mathematical Model}

To build the system's mathematical model, we first analyzed the mathematical model of the PMA before deriving a mathematical model of the shoulder, elbow, and wrist using a kinematic model (because of the similar derivation process, only the mathematical model of the shoulder is derived here). Given the elasticity, ductility, flexibility, toughness, and hysteresis of the PMA, we constructed a PMA static mathematical model as follows: ${ }^{(18)}$

$$
F=m(\varepsilon)\left(P_{0}+\Delta P\right)+h(\varepsilon)
$$

in which $m(\varepsilon)=k_{11}-k_{21} \varepsilon+k_{31} \exp (\mu \varepsilon), h(\varepsilon)=k_{12}-k_{22} \varepsilon+k_{32} \exp (\mu \varepsilon)$ where $k_{i 1}$ and $k_{i 2}$ are equation coefficients $(i=1,2), F$ is the muscle output force, $\varepsilon$ is the muscle contraction rate, $\mu$ is 


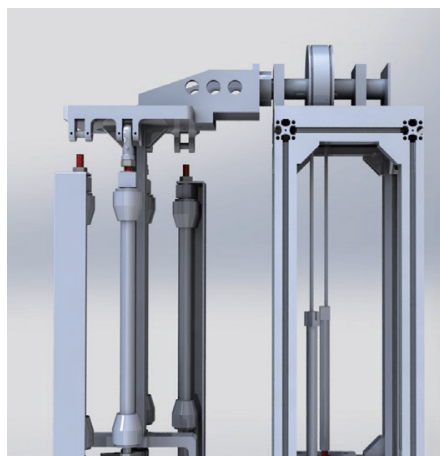

(a)

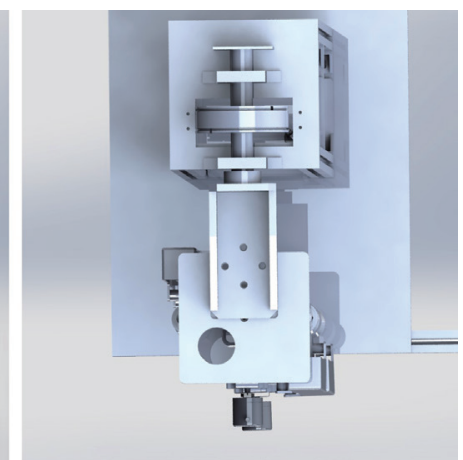

(b)

Fig. 3. (Color online) Shoulder joint of 6-DOF robotic manipulator. (a) Side view and (b) top view.

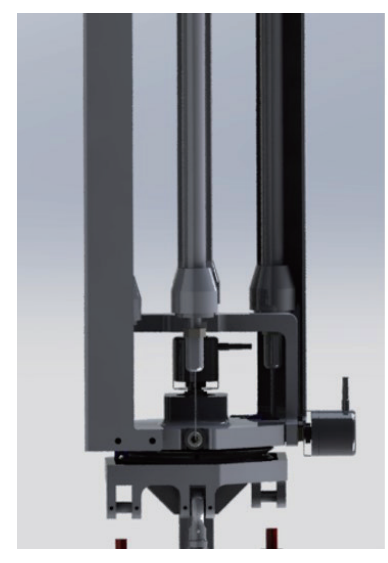

(a)

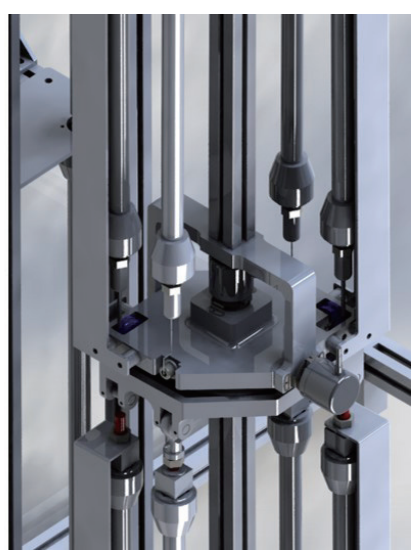

(b)

Fig. 4. (Color online) Elbow joint of 6-DOF robotic manipulator. (a) Side view and (b) oblique view.

the nonlinear attenuation coefficient of the contraction rate $\varepsilon, P_{0}$ is the prepressing force, and $\Delta P$ is the pressure variation volume. The PMA mathematical model in Eq. (1) is used to derive the joint's mathematical model; all joints use pairwise antagonism to drive rotation. The mathematical model of the shoulder is derived as follows:

$$
J_{S Y} \ddot{\theta}_{S Y}=\left(F_{S L}-F_{S R}\right) r_{S Y}-\left(M+\frac{m}{2}\right) g L_{\alpha} \sin \theta_{S Y}+d_{S Y} .
$$

The state variable is defined as $\left[x_{1}, x_{2}\right]=\left[\theta_{S Y}, \dot{\theta}_{S Y}\right]$, and the equation of back-and-forth motion of the shoulder is

$$
\left\{\begin{array}{l}
\dot{x}_{1}=x_{2} \\
\dot{x}_{2}=\frac{C_{3} r_{S Y}-C_{4} g L_{\alpha} \sin x_{1}}{J_{S Y}}+\frac{M_{S} r_{S Y}}{J_{S Y}} \Delta P_{S Y}+\frac{d_{S Y}}{J_{S Y}}, \\
y_{1}=x_{1}
\end{array}\right.
$$


in which $C_{3}=\left(M_{S} P_{0}+H_{S}\right), C_{4}=[M+(m / 2)], M_{S}=m\left(\varepsilon_{S L}\right)+m\left(\varepsilon_{S R}\right)$, and $H_{S}=h\left(\varepsilon_{S L}\right)+h\left(\varepsilon_{S R}\right)$. The above derivation procedure was also employed for the other joints.

\section{Kinematic Analysis}

Kinematic analysis is the foundation of path planning. Here, the variables of various axes of the manipulators are either converted into the terminal positions of the manipulators or used to plan the path of the terminal manipulator before joint variables are inversely derived. Therefore, kinematic analysis enables the robot manipulator to reach the target position with a smooth and safe path. The purpose of deriving the kinematic models is to determine the kinematic relations between the end-effector and the actuator motion of the robot and achieve trajectory tracking control. The correctness of the kinematic models is verified through the following steps. (i) Plan the desired spatial end-effector trajectory. (ii) Calculate the desired PMA trajectory by inverse kinematics using the data outputted from i. (iii) Calculate the position of the end-effector by forward kinematics using the information outputted from ii. (iv) Compare the calculated endeffector trajectory with the desired trajectory. The procedures are shown in Fig. 5. The kinematic analysis in both the forward and inverse directions and the workspace for the 6-DOF serial robot manipulator are described in the following.

\subsection{Forward kinematics}

The position of the shoulder rotary device is set as the origin of the world coordinates, and the parameters are established in Denavit-Hartenberg (D-H) parameters, as shown in Fig. 6, in accordance with the length of the connecting rod and a homogeneous transformation matrix, as shown in Table 1. The following is the $\mathrm{D}-\mathrm{H}$ homogeneous transformation matrix:

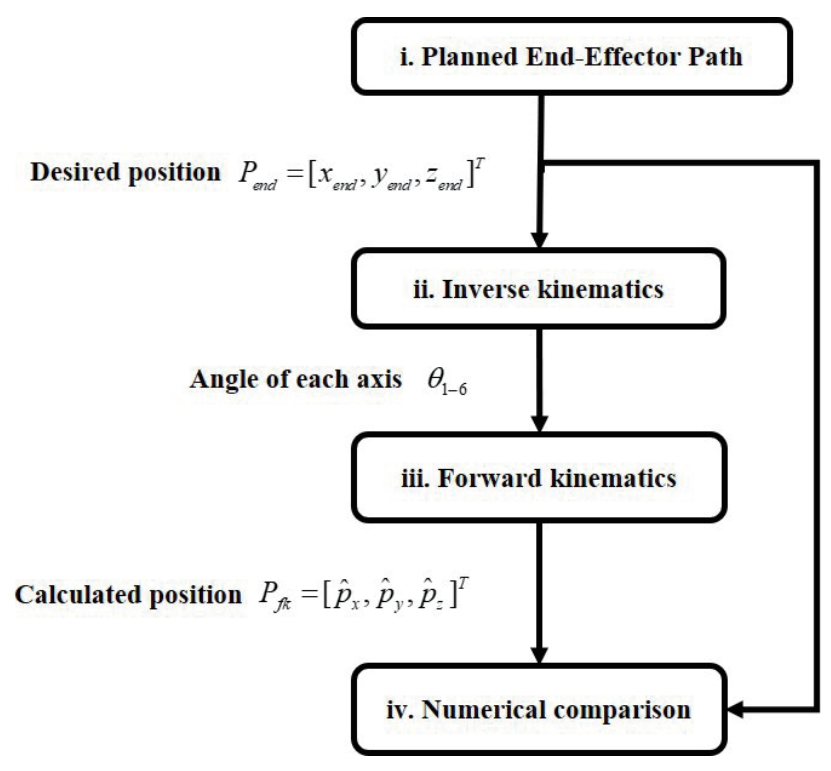

Fig. 5. Flow of kinematic verification. 


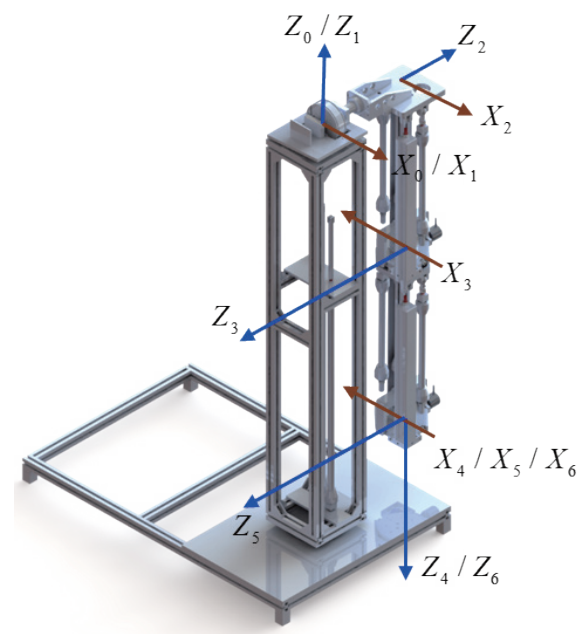

Table 1

D-H parameters.

\begin{tabular}{rrccc}
\hline$i$ & $\alpha_{i-1}$ & $a_{i-1}$ & $d_{i}$ & $\theta_{i}$ \\
\hline 1 & $0^{\circ}$ & 0 & 0 & $\theta_{1}$ \\
2 & $-90^{\circ}$ & 0 & $d_{2}$ & $\theta_{2}$ \\
3 & $180^{\circ}$ & $a_{2}$ & 0 & $\theta_{3}$ \\
4 & $-90^{\circ}$ & 0 & $d_{4}$ & $\theta_{4}$ \\
5 & $90^{\circ}$ & 0 & 0 & $\theta_{5}$ \\
6 & $-90^{\circ}$ & 0 & 0 & $\theta_{6}$ \\
\hline
\end{tabular}

Fig. 6. (Color online) D-H coordinate frames of the robotic manipulator.

$$
{ }_{i}^{i-1} T_{n}=\left[\begin{array}{cccc}
c \theta_{i} & -s \theta_{i} & 0 & a_{i} \\
s \theta_{i} c \alpha_{i-1} & c \theta_{i} c \alpha_{i-1} & -s \alpha_{i-1} & -s \alpha_{i-1} d_{i} \\
s \theta_{i} s \alpha_{i-1} & c \theta_{i} s \alpha_{i-1} & c \alpha_{i-1} & c \alpha_{i-1} d_{i} \\
0 & 0 & 0 & 1
\end{array}\right] .
$$

Here, $c=\cos \left(\theta_{i}\right), s=\sin \left(\theta_{i}\right)$, and $i=1, \ldots, 6$. After substituting the variables in the homogeneous transformation matrix with the coordinates of the connecting rod and the $\mathrm{D}-\mathrm{H}$ coordinates, the position of the end of the arm relative to the homogeneous matrix of the shoulder is found to be the following:

$$
{ }_{6}^{0} T_{n}={ }_{1}^{0} T_{n}{ }_{2}^{1} T_{n}{ }_{3}^{2} T_{n}{ }_{4}^{3} T_{n}{ }_{5}^{4} T_{n}{ }_{6}^{5} T_{n}=\left[\begin{array}{cccc}
\hat{n}_{x} & \hat{o}_{x} & \hat{a}_{x} & \hat{p}_{x} \\
\hat{n}_{y} & \hat{o}_{y} & \hat{a}_{y} & \hat{p}_{y} \\
\hat{n}_{z} & \hat{o}_{z} & \hat{a}_{z} & \hat{p}_{z} \\
0 & 0 & 0 & 1
\end{array}\right] .
$$

The equation of forward kinematics is

$$
\left\{\begin{array}{l}
\hat{p}_{x}=-a_{2} c_{1} s_{2}-d_{2} s_{1}+d_{4}\left(c_{1} c_{2} s_{3}-c_{1} s_{2} c_{3}\right) \\
\hat{p}_{y}=d_{2} c_{1}+d_{4}\left(c_{2} s_{1} s_{3}-c_{3} s_{1} s_{2}\right)-a_{2} s_{1} s_{2} \\
\hat{p}_{z}=-d_{4}\left(c_{2} c_{3}+s_{2} s_{3}\right)-a_{2} c_{2}
\end{array} .\right.
$$

To derive the workspace of the robot manipulator, shown in Figs. 7(a) and 7(b), Eq. (6) is applied. $P_{f k}=\left[\hat{p}_{x}, \hat{p}_{y}, \hat{p}_{z}\right]^{T}$ are substituted with the size of the robot manipulator's mechanism and the rotation angles of various joints. 


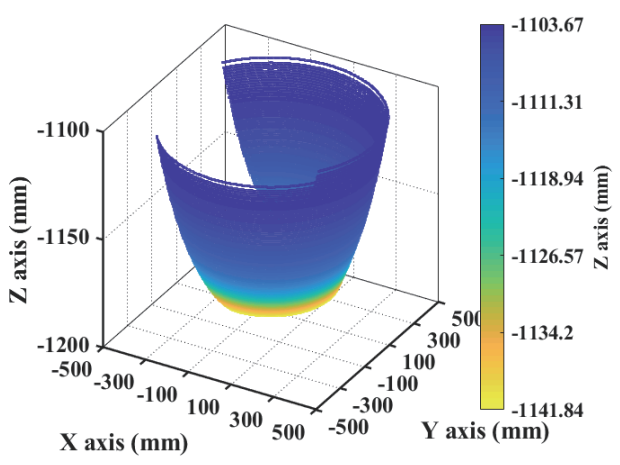

(a)

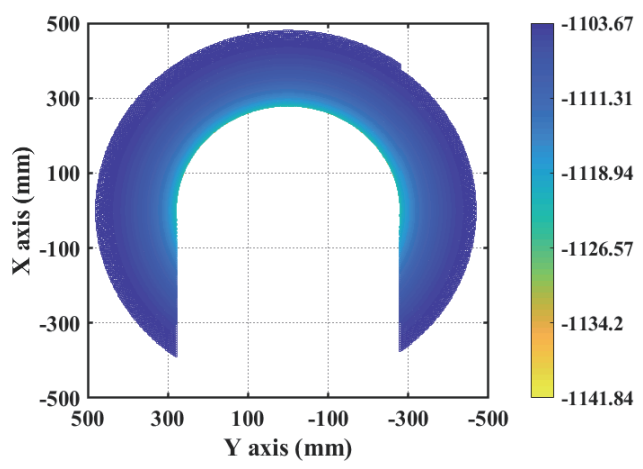

(b)

Fig. 7. (Color online) Workspace of robotic manipulator. (a) Isometric view and (b) top view.

\subsection{Inverse kinematics}

For pathing control of the robot manipulator, it is necessary to determine the path of the rotating angles of various joints based on the terminal manipulators' planned path via inverse kinematics. The following equation is derived from Eq. (6) and is used to determine the positions of the terminal manipulators:

$$
\hat{P}=\left[\begin{array}{c}
\hat{p}_{x} \\
\hat{p}_{y} \\
\hat{p}_{z}
\end{array}\right]=\left[\begin{array}{c}
-a_{2} c_{1} s_{2}-d_{2} s_{1}+d_{4}\left(c_{1} c_{2} s_{3}-c_{1} s_{2} c_{3}\right) \\
d_{2} c_{1}+d_{4}\left(c_{2} s_{1} s_{3}-c_{3} s_{1} s_{2}\right)-a_{2} s_{1} s_{2} \\
-d_{4}\left(c_{2} c_{3}+s_{2} s_{3}\right)-a_{2} c_{2}
\end{array}\right] .
$$

Based on the known positions during the movement of the terminal manipulators at $P_{\text {end }}=\left[x_{\text {end }}, y_{\text {end }}, z_{\text {end }}\right]^{T}$, the squares of the matrix elements in Eq. (7) are added to derive the following equation:

$$
{ }_{6}^{0} r^{2}=a_{2}^{2}+2 a_{2} d_{4} c \theta_{3}+d_{2}^{2}+d_{4}^{2}=x_{\text {end }}^{2}+y_{\text {end }}^{2}+z_{\text {end }}^{2} \text {. }
$$

Based on Eqs. (7) and (8), the angle of the third joint is set at

$$
\theta_{3}=2 \arctan \left( \pm \sqrt{\frac{{ }_{6}^{0} r^{2}-a_{2}^{2}-d_{2}^{2}-d_{4}^{2}-2 a_{2} d_{4}}{-2 a_{2} d_{4}-{ }_{6}^{0} r^{2}+a_{2}^{2}+d_{2}^{2}+d_{4}^{2}}}\right)
$$

Then $\theta_{3}$ is substituted for $\hat{p}_{z}$ to obtain

$$
\theta_{2}=2 \arctan \left( \pm \sqrt{\frac{z_{\text {end }}+a_{2}+d_{4} c_{3}}{-z_{\text {end }}-a_{2}+2 d_{4} s_{3}+d_{4}}}\right) .
$$


Finally, $\theta_{2}$ and $\theta_{3}$ are substituted for $\hat{p}_{x}$ to obtain

$$
\theta_{1}=2 \arctan \pm\left(\sqrt{\frac{x_{\text {end }}-d_{4} c_{2} s_{3}-d_{4} s_{2} c_{3}+a_{2} s_{2}}{-x_{\text {end }}-d_{4} c_{2} s_{3}-d_{4} s_{2} c_{3}+a_{2} s_{2}-2 d_{2}}}\right) .
$$

The Euler method is used to simplify the solution to

$$
{ }_{6}^{4} T=\left({ }_{1}^{0} T{ }_{2}^{1} T_{3}^{2} T{ }_{4}^{3} \operatorname{Rot}_{\hat{Z}_{4}}\left(-90^{\circ}\right)\right)^{-1},{ }_{6}^{0} T=\left[\begin{array}{lll}
r_{11} & r_{12} & r_{13} \\
r_{21} & r_{22} & r_{23} \\
r_{31} & r_{23} & r_{33}
\end{array}\right] .
$$

If $\theta_{5} \neq 0^{\circ}$, then $\theta_{4}=\arctan \left[r_{23} / s \theta_{5}, r_{13} / s \theta_{5}\right]$ and $\theta_{6}=\arctan \left[r_{32} / s \theta_{5},-r_{31} / s \theta_{5}\right]$. If $\theta_{5}=0^{\circ}$, then $\theta_{4}=0^{\circ}$ and $\theta_{6}=\arctan \left(-r_{12}, r_{11}\right)$. If $\theta_{5}=180^{\circ}$, then $\theta_{4}=0^{\circ}$ and $\theta_{6}=\arctan \left(r_{12},-r_{11}\right)$. Equations (8)-(12) are the inverse kinematic equations.

\section{Controller Design}

To avoid an excessive impact force in the case of a significant error, a controller was developed to ensure flexibility and safety. The controller enables the system to make a dynamic recovery via overdamping when problems such as sensor malfunction and pathing disruption occur. In the normal state, the controller can also adjust control signals for target pathing in accordance with the error.

\subsection{Novel proxy-based sliding mode control}

A LESO was designed to estimate the total disturbance of the system, with the status defined as $x=\left[x_{1}, x_{2}, x_{3}\right]^{T}=\left[\theta_{q}, \dot{\theta}_{q}, F\right]^{T}$, in which $\theta_{q}$ is the actual rotation angle, $\dot{\theta}_{q}$ is the actual rotation speed, and $F$ is the total disturbance. Therefore, Eq. (2) can be rearranged as

$$
\left\{\begin{array}{l}
\dot{x}_{1}=x_{2} \\
\dot{x}_{2}=x_{3}+b_{0} u(t) \\
\dot{x}_{3}=n(t)
\end{array}\right.
$$

in which $n(t)$ is the time derivative of $x_{3}$ and $b_{0}$ is a constant. The following LESO estimation system based on Eq. (13) is constructed:(19)

$$
\left\{\begin{array}{l}
\dot{\hat{x}}_{1}=\hat{x}_{2}-\beta_{1}\left(\hat{x}_{1}-x_{1}\right) \\
\dot{\hat{x}}_{2}=\hat{x}_{3}-\beta_{2}\left(\hat{x}_{1}-x_{1}\right)+b_{0} u(t), \\
\dot{\hat{x}}_{3}=-\beta_{3}\left(\hat{x}_{1}-x_{1}\right)
\end{array}\right.
$$


in which $\left[\hat{x}_{1}, \hat{x}_{2}, \hat{x}_{3}\right]^{T}=\left[\hat{\theta}_{q}, \dot{\hat{\theta}}_{q}, \hat{F}\right]^{T}$ is the observation system value of the observer and $\beta_{i}$ is the adjustable gain of the observer $(i=1,2,3)$. The following is the dynamic equation of the estimation error:

$$
\left\{\begin{array}{l}
\dot{\tilde{x}}_{1}=\dot{\hat{x}}_{1}-\dot{x}_{1}=\tilde{x}_{2}-\beta_{1} \tilde{x}_{1}, \\
\dot{\tilde{x}}_{2}=\dot{\hat{x}}_{2}-\dot{x}_{2}=\tilde{x}_{3}-\beta_{2} \tilde{x}_{1}, \\
\dot{\tilde{x}}_{3}=\dot{\hat{x}}_{3}-\dot{x}_{3}=-\beta_{3} \tilde{x}_{1}-n(t) .
\end{array}\right.
$$

Assumption. The boundary condition $n(t)>H$ holds, where $H>0$ is known.

Equation (15) can be expressed as follows:

$$
\dot{\tilde{\boldsymbol{x}}}=\boldsymbol{A} \tilde{\boldsymbol{x}}+\boldsymbol{D} n(t),
$$

in which $\tilde{\boldsymbol{x}}=\left[\begin{array}{lll}\tilde{x}_{1} & \tilde{x}_{2} & \tilde{x}_{3}\end{array}\right]^{T}, \boldsymbol{A}=\left[\begin{array}{ccc}-\beta_{1} & 1 & 0 \\ -\beta_{2} & 0 & 1 \\ -\beta_{3} & 0 & 0\end{array}\right]$, and $\boldsymbol{D}=\left[\begin{array}{lll}0 & 0 & -1\end{array}\right]^{T}$. Therefore, there exists a positive definite symmetric matrix $\boldsymbol{P}$ such that

$$
A^{T} P+P A=-Q
$$

for any given positive definite real symmetric matrix $\boldsymbol{Q}$. The following Lyapunov function is chosen:

$$
V_{L E S O}=\tilde{\boldsymbol{x}}^{\boldsymbol{T}} \boldsymbol{P} \tilde{\boldsymbol{x}}
$$

Equation (18) is then differentiated with respect to time:

$$
\dot{V}_{L E S O}=\dot{\tilde{\boldsymbol{x}}}^{\boldsymbol{T}} \boldsymbol{P} \tilde{\boldsymbol{x}}+\tilde{\boldsymbol{x}}^{\boldsymbol{T}} \boldsymbol{P} \dot{\tilde{\boldsymbol{x}}} .
$$

Finally, we rearrange the equation and derive the following inequality using Young's inequality:

$$
\dot{V}_{L E S O} \leq-\frac{\left(\lambda_{\min }(\boldsymbol{Q})-H\right)}{\lambda_{\max }(\boldsymbol{P})} V_{L E S O}+H\|\boldsymbol{P}\|^{2},
$$

in which $\lambda_{\min }(\boldsymbol{Q})$ is the minimum characteristic value of matrix $\boldsymbol{Q}$ and $\lambda_{\max }(\boldsymbol{P})$ is the maximum characteristic value of matrix $\boldsymbol{P}$. According to Eq. (20), when $H\|\boldsymbol{P}\|^{2}$ has a boundary and $\left(\lambda_{\text {min }}(\boldsymbol{Q})-H\right)>0$, with the suitable adjustment of $\beta_{1}, \beta_{2}$, and $\beta_{3}, \dot{V}_{L E S O}<0$. Therefore, with the gradual stabilization of $\tilde{\boldsymbol{x}}(t)$, the estimated state in Eq. (15) will converge gradually to the actual value. 
We propose a novel proxy-based sliding mode controller (NPSMC) scheme that can produce slow, overdamped resuming motion after actuator-force saturation without sacrificing accurate, responsive tracking capability during normal operations. A physical interpretation of the scheme is illustrated in Fig. 8. The concept of an NPSMC is to add a proxy object between the actual controlled object and the expected position. The sliding mode controller controls the proxy and it tracks the expected position. Furthermore, the proxy links the real controlled object with a virtual coupling, which can be regarded as a PID controller of a spring system; this enables the actual controlled object to track the proxy object via the PID controller. Finally, we define $\left[\theta_{d}, \dot{\theta}_{d}\right]$ as the expected angle and expected angular velocity, with $\left[\theta_{p}, \dot{\theta}_{p}\right]$ as the angle and angular velocity of the proxy-based virtual object, respectively. To obtain a stable extended PSMC for the nonlinear system in Eq. (3), we design the following novel sliding manifolds:

$$
\begin{gathered}
s_{p}=\dot{\theta}_{d}-\dot{\theta}_{p}+\lambda_{H}\left(\theta_{d}-\theta_{p}\right), \\
s_{q}=\dot{\theta}_{d}-\dot{\hat{\theta}}_{q}+\lambda_{H}\left(\theta_{d}-\hat{\theta}_{q}\right),
\end{gathered}
$$

where $\lambda_{H}$ is a positive integer. Furthermore, we also design a virtual coupling force $\tau_{N c o u}$ and sliding mode controller $\tau_{N s m c}$ as

$$
\begin{gathered}
\tau_{N c o u}=\frac{1}{b_{0}}\left[-\hat{F}+K_{p}\left(\theta_{p}-\hat{\theta}_{q}\right)+K_{d}\left(\dot{\theta}_{p}-\dot{\hat{\theta}}_{q}\right)+\lambda_{H}\left(\dot{\theta}_{d}-\dot{\hat{\theta}}_{q}\right)+\ddot{\theta}_{d}\right], \\
\tau_{N s m c}=\gamma_{N} \operatorname{sgn}\left(s_{p}\right)-K_{p}\left(\theta_{p}-\hat{\theta}_{q}\right)+J_{p} \ddot{\theta}_{d}+J_{p} \lambda_{H}\left(\dot{\theta}_{d}-\dot{\theta}_{p}\right)-K_{d}\left(\dot{\theta}_{p}-\dot{\hat{\theta}}_{q}\right)+\tau_{N c o u},
\end{gathered}
$$

where the total disturbance $\hat{F}$ is designed with the LESO and $\lambda_{N}$ is a positive real number, while $K_{p}$ and $K_{d}$ are the proportional and differential gains, respectively. Note that the proposed virtual coupling force $\tau_{N c o u}$ is not a pure PI-type controller. Some terms based on known dynamics are added for the convenience of theoretical analysis. A similar consideration is taken in the design

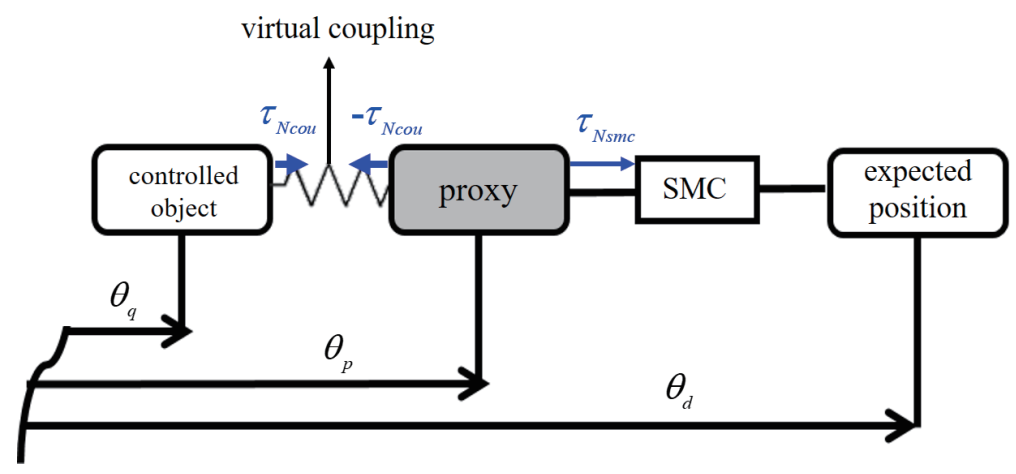

Fig. 8. (Color online) Physical interpretation of NPSMC. 
of the sliding mode controller. $J_{p}$ is the rotational inertia of the virtual object and $\tau_{N s m c}$ and $\tau_{N c o u}$ are active forces imposed on the proxy virtual object. The virtual object has hypothetical inertia of $J_{p}=0$. Hence, the following equation of motion was obtained on the basis of Newton's second law of motion:

$$
J_{P} \ddot{\theta}_{p}=\tau_{N s m c}-\tau_{N c o u},
$$

which can be expressed as

$$
\dot{\theta}_{p}+S_{N}=\frac{\gamma_{N}}{K_{d}} \operatorname{sgn}\left[\dot{\theta}_{d}+\lambda_{H}\left(\theta_{d}-\theta_{p}\right)\right]
$$

where $S_{N}=\left[K_{P}\left(\theta_{p}-\hat{\theta}_{q}\right)-K_{d} \dot{\hat{\theta}}_{q}\right] / K_{d}$. The $\operatorname{sgn}(\cdot)$ and $\operatorname{sat}(\cdot)$ have equivalent variations, ${ }^{(7)}$ with $\operatorname{sgn}(\cdot)$ replaced by sat( $(\cdot)$ in Eq. (26) to solve the problem of high-frequency chattering. Then Eq. (26) can be rewritten as

$$
\dot{\theta}_{p}+S_{N}=\frac{\gamma_{N}}{K_{d}} \operatorname{sat}\left(\frac{\dot{\theta}_{d}+\lambda_{H}\left(\theta_{d}-\theta_{p}\right)+S_{N}}{\frac{\gamma_{N}}{K_{d}}}\right) .
$$

Using Eq. (23) to describe the controller for the controlled object, we substitute Eq. (27) for Eq. (23) then rearrange to derive the control law. We define $a=\theta_{p}-\hat{\theta}_{q}, e=\theta_{d}-\hat{\theta}_{q}$.

$$
\tau_{N P S M C}=\frac{1}{b_{0}}\left[\lambda_{H} \dot{e}+\ddot{\theta}_{d}-\hat{F}+\gamma_{N} \operatorname{sat}\left(\frac{1}{\gamma_{N}}\left(K_{d} s_{q}-\left(K_{d} \lambda_{H}-K_{p}\right) a\right)\right)\right]
$$

In accordance with Ref. 9, the PSMC control output $\tau_{N P S M C}$ can be directly replaced with $\triangle P_{N P S M C}$, because the joint output torque that is driven by pneumatic muscles is associated with the pressure difference of a pair of PMAs. Since the NPSMC is based on a PSMC, the NPSMC can also be replaced with $\triangle P_{N P S M C}$, resulting in the following:

$$
\Delta P_{N P S M C}=\frac{1}{b_{0}}\left[\lambda_{H} \dot{e}+\ddot{\theta}_{d}-\hat{F}+\gamma_{N} \operatorname{sat}\left(\frac{1}{\gamma_{N}}\left(K_{d} s_{q}-\left(K_{d} \lambda_{H}-K_{p}\right) a\right)\right)\right] .
$$

\subsection{Stability analysis}

To analyze the stability of the NPSMC, we first define the system estimation status as $\left[\hat{x}_{1}, \hat{x}_{2}, \hat{x}_{3}\right]^{T}=\left[\hat{\theta}_{q}, \hat{\theta}_{q}, \hat{F}\right]^{T}$; with $\boldsymbol{x}_{e}=\left[s_{p}, s_{q}, \theta_{p}-\hat{x}_{1}\right]$ as the system's balance point. Then we select the following Lyapunov functions: ${ }^{(20)}$ 


$$
\begin{gathered}
V=V_{1}+V_{2}, \\
V_{1}=\frac{1}{2} J_{p} s_{p}^{2}+\frac{1}{2} s_{q}^{2}, \\
V_{2}=\frac{1}{2}\left(K_{p}+K_{d} \lambda_{H}\right)\left(\theta_{p}-\hat{x}_{1}\right)^{2} .
\end{gathered}
$$

The Lyapunov function of Eq. (30) can be satisfied with

$$
\begin{cases}V>0, & \boldsymbol{x}_{e} \neq \mathbf{0} \\ V=0, & \boldsymbol{x}_{\boldsymbol{e}}=\mathbf{0}\end{cases}
$$

Differentiating both sides of Eq. (31) with respect to time gives

$$
\dot{V}_{1}=J_{p} s_{p} \dot{s}_{p}+s_{q} \dot{s}_{q}
$$

Equations (21)-(23) and (25) are substituted into Eq. (34) to obtain

$$
\begin{aligned}
\dot{V}_{1} & =s_{p}\left[J_{p} \ddot{\theta}_{d}-J_{p} \ddot{\theta}_{p}+J_{p} \lambda_{h}\left(\dot{\theta}_{d}-\dot{\theta}_{p}\right)\right]+s_{q}\left[\ddot{\theta}_{d}-\hat{x}_{3}+\lambda_{H}\left(\dot{\theta}_{d}-\hat{x}_{2}\right)\right] \\
& =s_{p} s_{p 1}+s_{q}\left[\ddot{\theta}_{d}-\dot{x}_{2}-\tilde{x}_{3}+\lambda_{H}\left(\dot{\theta}_{d}-\hat{x}_{2}\right)\right] \\
& =s_{p} s_{p 1}+s_{q}\left[-K_{p}\left(\theta_{p}-\hat{x}_{1}\right)-K_{d}\left(\dot{\theta}_{p}-\hat{x}_{2}\right)\right] \\
& =-\gamma_{N}\left|s_{p}\right|-K_{p}\left(\theta_{p}-\hat{x}_{1}\right)\left(\dot{\theta}_{p}-\hat{x}_{2}\right)-K_{d} \lambda_{H}\left(\theta_{p}-\hat{x}_{1}\right)\left(\dot{\theta}_{p}-\hat{x}_{2}\right)-K_{p} \lambda_{H}\left(\theta_{p}-\hat{x}_{1}\right)^{2}-K_{d}\left(\dot{\theta}_{p}-\hat{x}_{2}\right)^{2} .
\end{aligned}
$$

Similarly to above, we obtain

$$
\begin{aligned}
\dot{V}_{2} & =\left(K_{p}+K_{d} \lambda_{H}\right)\left(\theta_{p}-\hat{x}_{1}\right)\left(\dot{\theta}_{p}-\hat{x}_{2}\right) . \\
& =K_{p}\left(\theta_{p}-\hat{x}_{1}\right)\left(\dot{\theta}_{p}-\hat{x}_{2}\right)+K_{d} \lambda_{h}\left(\theta_{p}-\hat{x}_{1}\right)\left(\dot{\theta}_{p}-\hat{x}_{2}\right) .
\end{aligned}
$$

Using Eqs. (35) and (36), we obtain the differential of the Lyapunov function as follows:

$$
\begin{aligned}
\dot{V} & =\dot{V}_{1}+\dot{V}_{2} \\
& =-\gamma_{N}\left|s_{p}\right|-K_{p} \lambda_{H}\left(\theta_{p}-\hat{x}_{1}\right)^{2}-K_{d}\left(\dot{\theta}_{p}-\hat{x}_{2}\right)^{2} \leq 0 .
\end{aligned}
$$

As shown from Eqs. (31) and (32), $V \geq 0$, while $\dot{V} \leq 0$. According to the stability criterion, the system is consistently stable at the equilibrium point $x_{e}=\left[s_{p}, s_{q}, \theta_{p}-\hat{x}_{1}\right]=0$. Figure 9 shows the flow of the control scheme of the system. Figure 10 shows the overall control block of the 6-DOF robot manipulator. The 6-DOF robot manipulator is implemented by using distributed control, 


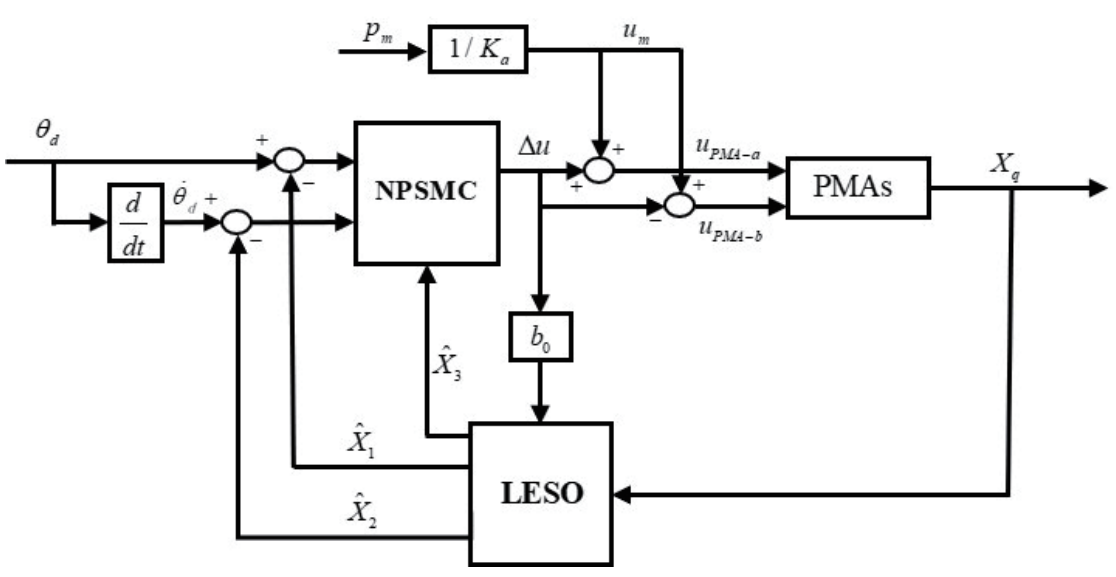

Fig. 9. Control scheme of NPSMC for the PMAs.

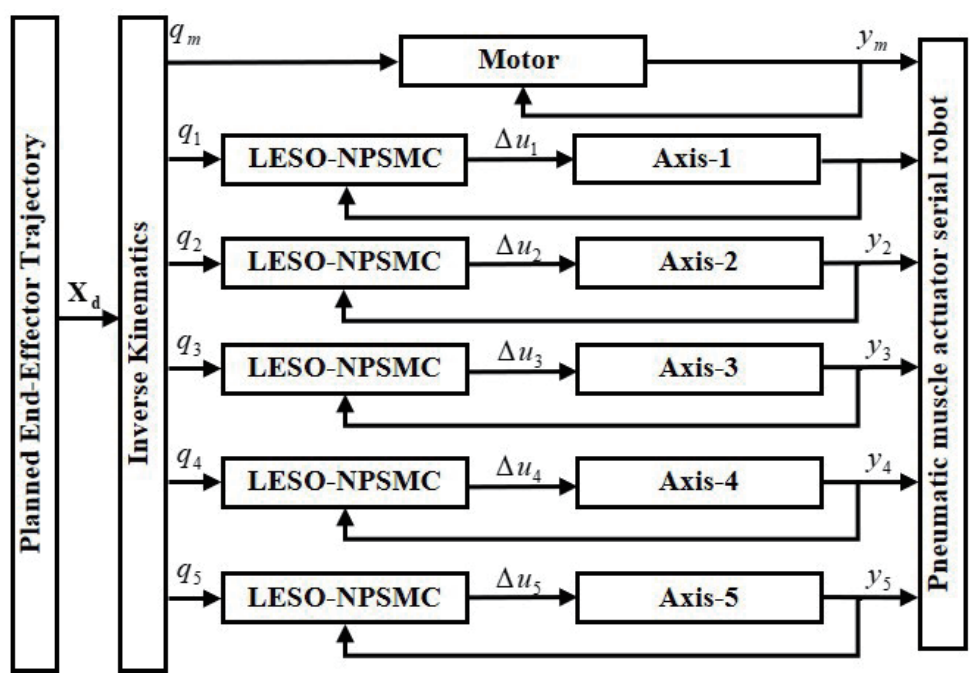

Fig. 10. Overall control scheme of robotic manipulator.

meaning that forces from other joints are considered as external disturbances when designing the LESO-NPSMC for joints. In Fig. 10, $\boldsymbol{X}_{\boldsymbol{d}}$ is the reference coordinate, $q_{m}$ is the desired angle, $q_{i}$ $(i=1, \ldots, 5)$ are the desired displacements, $\Delta u_{i}(i=1, \ldots, 5)$ are the variations of the control force, and $y_{m}$ and $y_{i}(i=1, \ldots, 6)$ are the system outputs.

\section{Experimental Results}

The NPSMC strategy was experimentally tested using the 6-DOF robot manipulator shown in Fig. 1, which was controlled through a cRIO-9040 system that is embedded in the control loop to achieve real-time control. It receives all measurement data via interface cards and sends an input voltage command to proportional pressure regulating valves. The interface cards include an NI-9411 digital I/O converter, an NI-9264 analog output interface card, and an NI-9203 analog input interface card. An industrial PC provides a LabVIEW integrated development environment (IDE) for developers and downloads programs into the cRIO-9040 system. In this 
paper, each joint of the 6-DOF robot manipulator is treated as an independent system. Moreover, the effects of one system on another are considered as uncertainties in the controller design. To overcome the uncertainties and external load forces, an NPSMC strategy is used for each joint. All parameters of the controllers are designed by experts and tuned by trial and error. The sampling interval of the controllers was $T=0.001 \mathrm{~s}$. The PID gains were chosen by trial and error to realize as accurate and stiff angle control as possible. This trial-and-error gain tuning was performed in the same manner as that for the PID control because the NPSMC is equivalent to the traditional PID control when the actuators are not saturated.

\subsection{Set-point control}

A set of experiments was performed to show the resuming motion from significant angular errors, which can be produced when discontinuous desired angles are provided as set-point control with large external forces. The solid blue line in Fig. 11(a) shows the desired angles that are discontinuously changed. The formulation is

$$
y_{\text {elbow }}(t)= \begin{cases}15, & 0 \leq t<7.5 \\ 0, & 7.5 \leq t<15,\end{cases}
$$
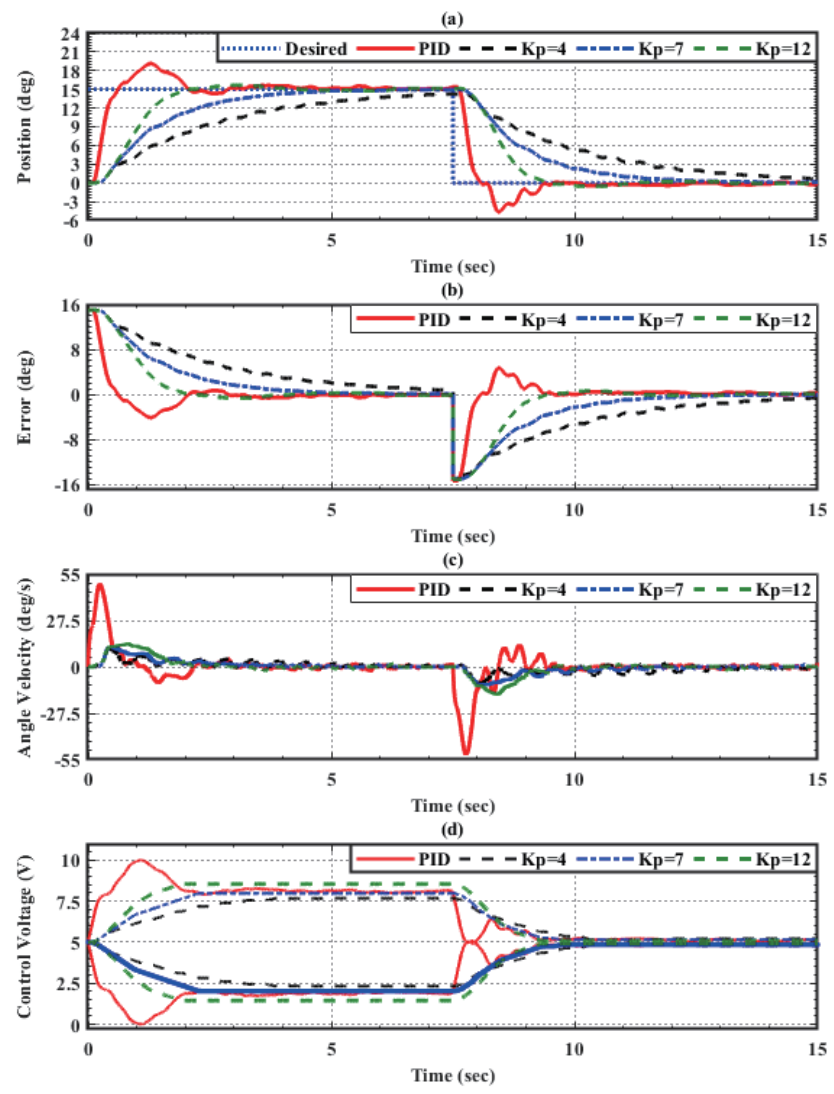

Fig. 11. (Color online) Shoulder joint compliance test. (a) Pathing response, (b) control error, (c) angle velocity, and (d) control voltage. 
where $y_{\text {elbow }}$ is the elbow joint angle. The results of the NPSMC and PID control for the elbow joint are shown in Fig. 11. The results clearly show that the NPSMC effectively removes overshoots and oscillations produced with PID control and exhibits overdamped, exponential resuming motion toward the desired angles. The PID control may or may not succeed in suppressing overshoots and oscillation, and its resultant motion strongly depends on the controlled objects. In contrast, the NPSMC always produces exponential motion.

\subsection{Path-tracking control for end-effector}

In this section, an experiment on the proposed NPSMC is implemented with the pathtracking control of the end-effector. The desired trajectory is shown in Fig. 12. The solid blue lines in Figs. 13(a), 14(a), and 15(a) show the desired trajectories of the shoulder, elbow, and wrist joints calculated by the inverse kinematics, respectively. The path-tracking experimental results of the shoulder, elbow, and wrist joints corresponding to the end-effector's required trajectory in Fig. 12 are shown in Figs. 13-15, respectively. Figure 16 shows the overall tracking response of the end-effector's required trajectory in Fig. 12. The maximum absolute error of the shoulder, elbow, and wrist joints, the maximum error in the Euclidian spatial distance of the end-effector, and the mean square error are used for performance evaluation. The error in the Euclidian spatial distance for the coordinate system is expressed as

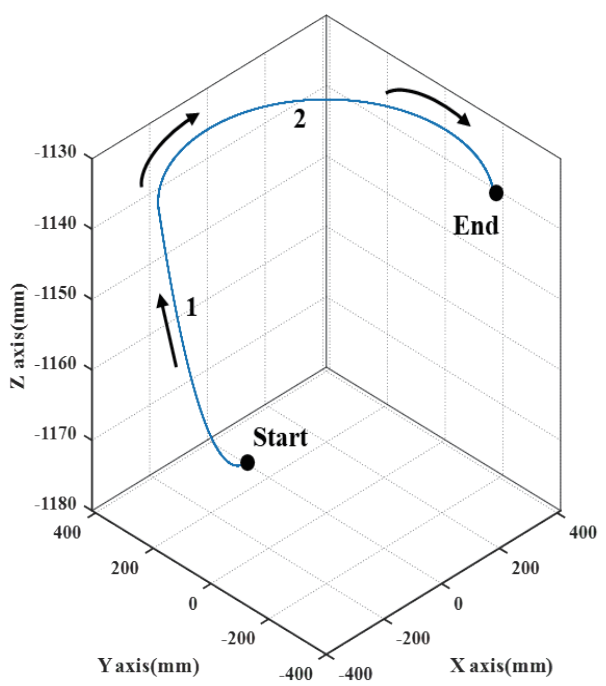

Fig. 12. (Color online) Designed trajectory for path tracking control experiment.
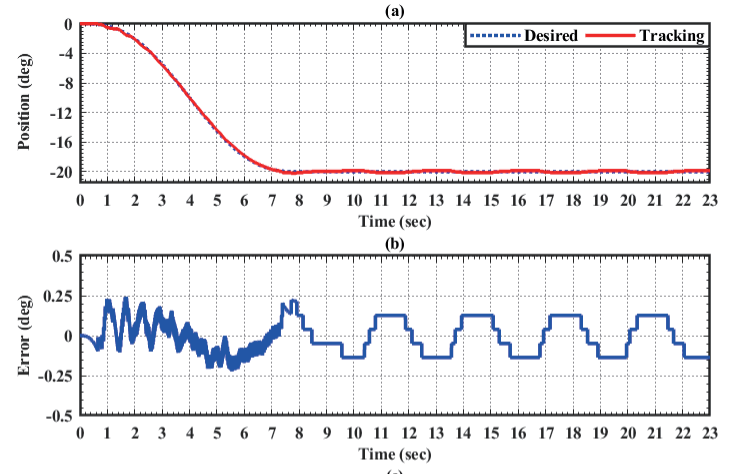
(c)
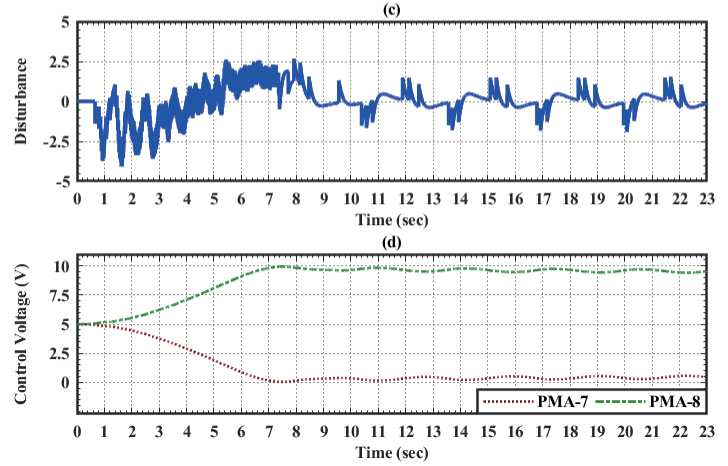

Fig. 13. (Color online) Experimental results of shoulder path-tracking. (a) Pathing response, (b) system error, (c) system disturbance, and (d) control voltage. 

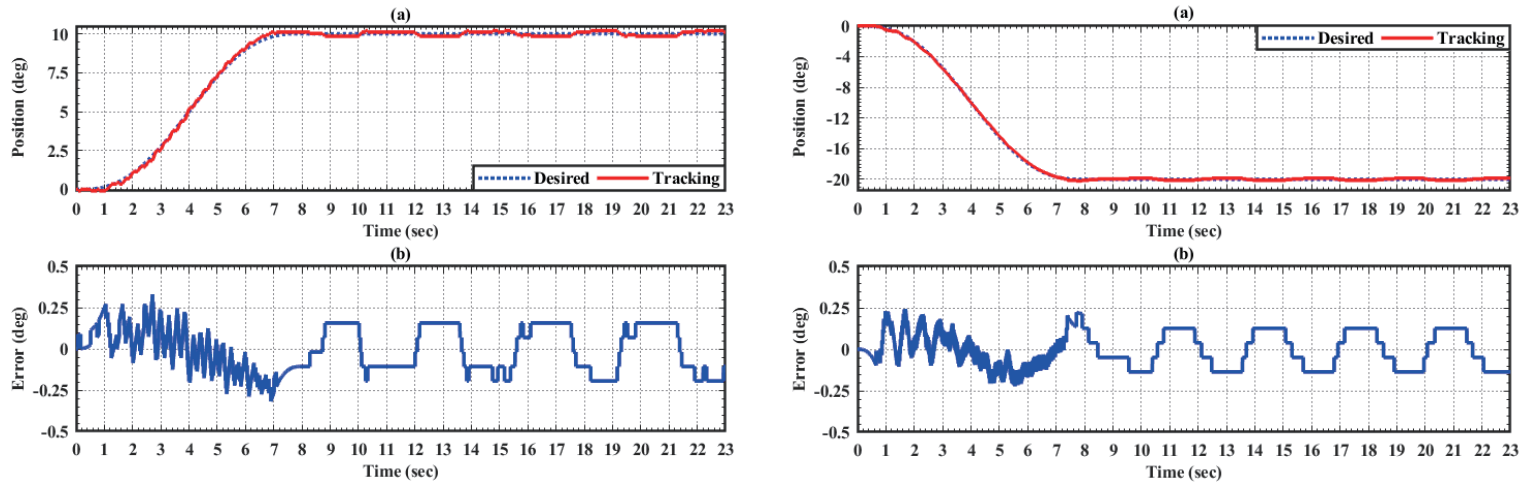

(c)
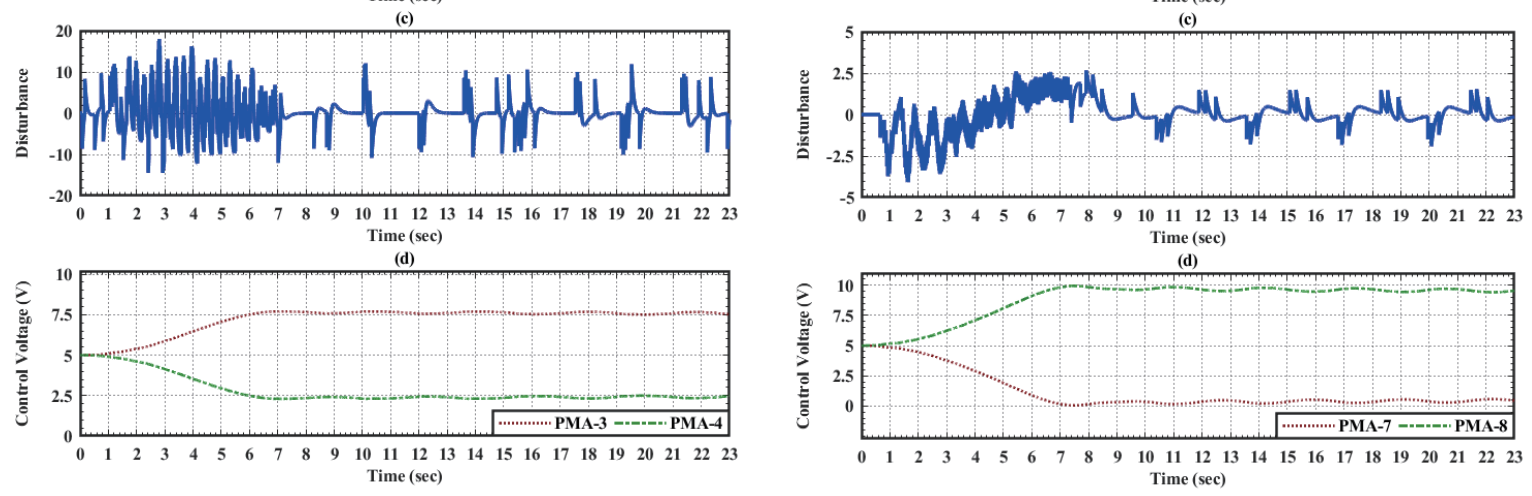

Fig. 14. (Color online) Experimental results of elbow path-tracking. (a) Pathing response, (b) system error, (c) system disturbance, and (d) control voltage.

Fig. 15. (Color online) Experimental results of wrist path-tracking. (a) Pathing response, (b) system error, (c) system disturbance, and (d) control voltage.

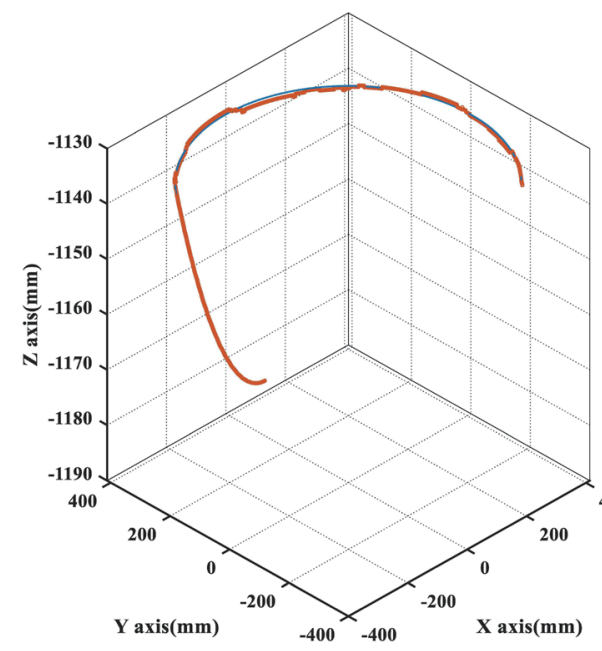

Fig. 16. (Color online) Experimental results of end-effector path-tracking.

$$
e_{\text {end-effector }}=\sqrt{e_{x_{p}}^{2}+e_{y_{p}}^{2}+e_{z_{p}}^{2}}
$$

where $e_{x_{p}}=x_{p, d}-x_{p}, e_{y_{p}}=y_{p, d}-y_{p}$, and $e_{z_{p}}=z_{p, d}-\mathrm{z}_{p}$ are the errors between the desired endeffector position $\left(x_{p, d}, y_{p, d}, z_{p, d}\right)$ and the calculated end-effector position with respect to the angle 


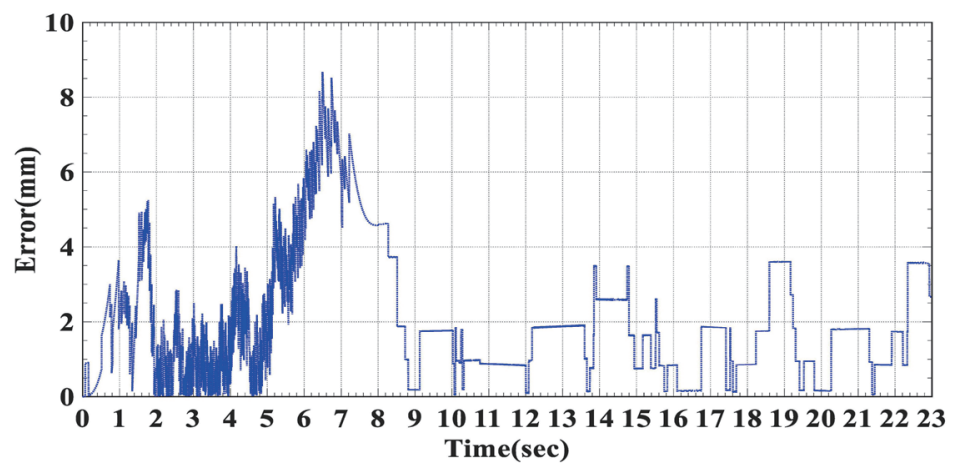

Fig. 17. (Color online) Experimental results of end-effector path-tracking error.

Table 2

Performance of 6-DOF robot manipulator for the pathing trajectory.

\begin{tabular}{lccc}
\hline & Figure & $\begin{array}{c}\text { Max. absolute error } \\
(\mathrm{mm})\end{array}$ & $\begin{array}{c}\text { Mean-square error } \\
\left(\mathrm{mm}^{2}\right)\end{array}$ \\
\hline End-effector & Fig. 13 & 8.6751 & 6.8802 \\
Shoulder joint & Fig. 16 & 0.3302 & 0.0194 \\
Elbow joint & Fig. 17 & 0.3082 & 0.0080 \\
Wrist joint & Fig. 18 & 0.2435 & 0.0117 \\
\hline
\end{tabular}

of each joint of the 6-DOF robot manipulator obtained by the inverse kinematics. The estimated position error of the end-effector is calculated from the angle tracking error of the actuated joint, as shown in Fig. 17. Table 2 indicates the total performance of the 6-DOF robot manipulator for the pathing trajectory. For the NPSMC, each joint's maximum absolute error is under $0.34 \mathrm{~mm}$ and the spatial error of the end-effector is under $8.7 \mathrm{~mm}$.

\section{Conclusions}

The PMA is promising because it is essentially a soft actuator. Unlike motors, the PMA is driven by compressed air and can provide necessary compliance for robotic manipulators. We used pneumatic technology and a PMA made of special materials to improve the safety of 6-DOF manipulators. However, because of its nonlinearities, time-varying parameters, and hysteresis, it is not easy to control accurately. Conventional control strategies, such as sliding mode control, suffer from the "chattering" problem. Hence, a novel LESO-based NPSMC strategy is proposed and validated in a real 6-DOF robot manipulator. The stability of the NPSMC system is proven using the Lyapunov theorem, and the strong conjecture assumed in the traditional PSMC is then avoided. Finally, the functions and the safety performance of the NPSMC were verified in an experiment on the manipulator, demonstrating its improved safety and excellent pathing tracking performance. Further applications of the PMA in a manipulator system will be explored in future studies. 


\section{Acknowledgments}

This work was supported by the Ministry of Science and Technology, Taiwan, R.O.C., under Grants No. MOST 109-2622-E-005-006-CC3, MOST 108-2628-E-005-003-MY2, and MOST 107-2221-E-005-077.

\section{References}

1 D. E. Whitney: IEEE Trans. Man-Mach Syst. 10 (1969) 47. https://doi.org/10.1109/TMMS.1969.299896

2 P. Ohta, L. Valle, J. King, K. Low, J. Yi, C. G. Atkeson, and Y. L. Park: Soft Rob. 5 (2018) 204. http://doi. org/10.1089/soro.2017.0044

3 N. Hogan: ASME J. Dyn. Syst. Meas. Control. 107 (1985) 1. https://doi.org/10.1115/1.3140713

4 J. Duan, Y. Gan, M. Chen, and X. Dai: Rob. Auton. Syst. 102 (2018) 54. https://doi.org/10.1016/j. robot.2018.01.009

5 J. Koivumäki and J. Mattila: IEEE/ASME Trans. Mechatron. 22 (2016) 601. https://doi.org/10.1109/ TMECH.2016.2618912

6 P. H. A. Ho, V. K. Cao, and T. N. Nguyen: Robotica. 36 (2018) 1333. https://doi.org/10.1017/S0263574718000450

7 R. Kikuuwe, S. Yasukouchi, H. Fujimoto, and M. Yamamoto: IEEE Trans. Rob. 26 (2010) 670. https://doi. org/10.1109/TRO.2010.2051188

8 G. Byun and R. Kikuuwe: ROBOMECH J. 7 (2020) 1. https://doi.org/10.1186/s40648-020-00166-1

9 M. Van Damme, B. Vanderborght, B. Verrelst, R. Van Ham, F. Daerden, and D. Lefeber: Int. J. Rob. Res. 28 (2009) 266. https://doi.org/10.1177/0278364908095842

10 E. Çandır: Cascaded Proxy-Based Sliding Mode Control Enhanced with Disturbance Observer for the Stabilization and Control of a Gun-Turret Platform (METU, Ankara, 2014) Chap. 5.

11 R. Kikuuwe: IMA J. Math. Control Inf. 35 (2018) 1319. https://doi.org/10.1093/imamci/dnx030

12 G. Ding, J. Huang, B. Hu, and Z.-H. Guan: Proc. 2017 11th Asian Control Conf. (ASCC, 2017) 2917-2922. https://doi.org/10.1109/ASCC.2017.8287641

13 J. Han: IEEE Trans. Ind. Electron. 56 (2009) 900. https://doi.org/10.1109/TIE.2008.2011621

14 Z. Gao: Proc. 2003 the American Control Conf. (ACC, 2003) 4989-4996. https://doi.org/10.1109/ ACC.2003.1242516

15 D. Yoo, S. T. Yau, and Z. Gao: Int. J. Control 80 (2007) 102. https://doi.org/10.1080/00207170600936555

16 Z. Gao, S. Hu, and F. Jiang: Proc. 40th IEEE Conf. Decision and Control (CDC, 2001) 4877-4882. https://doi. org/10.1109/CDC.2001.980980

17 Q. Zheng, Z. Chen, and Z. Gao: Control Eng. Pract. 17 (2009) 1016. https://doi.org/10.1016/j. conengprac.2009.03.005

18 H. Yu, W. Guo, H. Tan, M. Li, and H. Cai: J. Mech. Eng. 48 (2012) 1. https://doi.org/10.3901/JME.2012.17.00

19 W. Zhao, A. Song, and Y. Cao: Appl. Sci. 9 (2019) 1571. https://doi.org/10.3390/app9081571

20 Y. T. Liu, T. T. Kung, K. M. Chang, and S. Y. Chen: Precis. Eng. 37 (2013) 522. https://doi.org/10.1016/j. precisioneng.

\section{About the Authors}

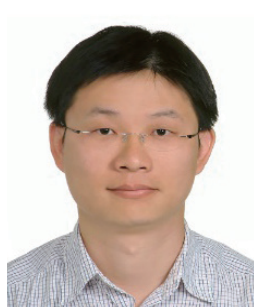

Lian-Wang Lee received his M.S. and Ph.D. degrees in automation and control from National Taiwan University of Science and Technology, Taipei, Taiwan, R.O.C., in 2000 and 2009, respectively. From 2002 to 2006, he was an assistant researcher in the electronic system research division of National Chung-Shan Institute of Science and Technology. From 2010 to 2018, he was an assistant professor and associate professor with the Department of Mechanical Engineering, Lunghwa University of Science and Technology, 


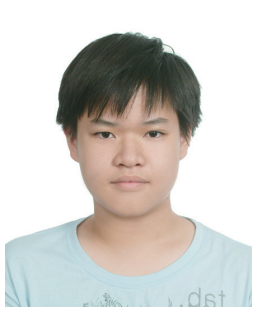

Taoyuan City, Taiwan. Now he is an associate professor with the Department of Mechanical Engineering, National Chung Hsing University, Taichung, Taiwan. His current research interests are in the areas of fluid power control, intelligent systems and control, mechatronics, vehicle dynamics and control, and robot control. (leelw@dragon.nchu.edu.tw)

Liang-Yu Lu received a B.S. degree from National Chin-Yi University of Technology, Taiwan, in 2020 and is currently studying for a master's degree in the Heat Flow Group of the Department of Mechanical Engineering, National Sun Yat-sen University. (3a516108@gm.student.ncut.edu.tw)

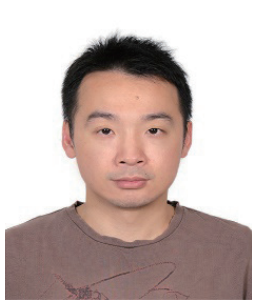

I-Hsum Li received his Ph.D. degree in electrical engineering from National Taiwan University of Science and Technology, Taipei, Taiwan, in 2007. From 2007 to 2008, he worked as a postdoctoral researcher of National Taiwan University of Science and Technology and National Taipei University of Science and Technology. In 2009, he became an assistant professor of the Department of Information Technology, Lee-Ming Institute of Technology, where he became an associate professor in 2012. In 2017, he started work as an associate professor of the Department of Electrical Engineering of Chinese Culture University. Now he is an associate professor of the Department of Mechanical and Electro-Mechanical Engineering, Tamkang University, New Taipei City, Taiwan. His current research interests are in the areas of intelligent systems, vehicle dynamics and control, and robot control.

(ihsumlee@gmail.com)

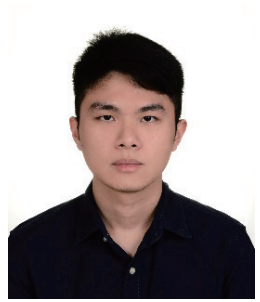

Chia-Wei Lee received his B.S. degree in mechanical engineering from Tatung University, Taipei, Taiwan, R.O.C., in 2019. Now he is a master's student in the Department of Mechanical Engineering at National Chung Hsing University, Taichung, Taiwan, R.O.C. His current research interests are in the areas of pneumatic robotic arms and fractional-order control. (tommywoei@gmail.com)

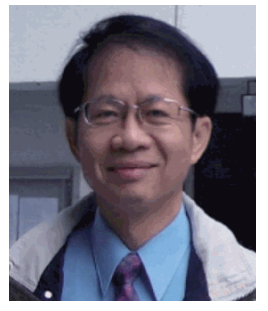

Te-Jen Su received his Ph.D. degree in electrical engineering from National Cheng-Kung University Tainan, Taiwan, in 1989. He is currently a professor with the Department of Electronic Engineering, National Kaohsiung University of Sciences and Technology, Kaohsiung City, Taiwan, R.O.C., and Kaohsiung Medical University. His research interests include intelligent control systems, embedded processor design, and satellite communication systems. (sutj@nkust.edu.tw) 